\title{
On a certain way of proving the solvability for boundary value problems
}

\section{Arnold Y Lepin}

${ }^{*}$ Correspondence: ctr34@inbox.lv Institute of Mathematics and Computer Science, University of Latvia, Riga, Latvia

\begin{abstract}
A certain way of replacing a given boundary value problem by another one, a solution of which solves also the original problem, is considered.
\end{abstract}

MSC: $34 \mathrm{~B} 15$

Keywords: boundary value problems; upper and lower functions

Consider the solvability of the boundary value problem (BVP)

$$
\begin{array}{ll}
\left(\varphi\left(t, x, x^{\prime}\right)\right)^{\prime}=f\left(t, x, x^{\prime}\right), & t \in I=[a, b], \\
H_{1} x=h_{1}, \quad H_{2} x=h_{2}, & \alpha \leq x \leq \beta,
\end{array}
$$

where $\varphi \in C\left(I \times R^{2}, R\right)$ is strictly increasing in $x^{\prime}$ for fixed $t$ and $x, f: I \times R^{2} \rightarrow R$ satisfies the Caratheodory conditions, that is, $f(t, \cdot, \cdot)$ is measurable in $I$ for fixed $x, x^{\prime} \in R, f\left(\cdot, x, x^{\prime}\right)$ is continuous on $R^{2}$ for fixed $t \in I$, and for any compact set $P \subset R^{2}$ there exists function $g \in L(I, R)$ such, that for any $\left(t, x, x^{\prime}\right) \in I \times P$, the estimate $\left|f\left(t, x, x^{\prime}\right)\right| \leq g(t)$ holds, $H_{1}, H_{2} \in$ $C\left(C^{1}(I, R), R\right), h_{1}, h_{2} \in R, \alpha$ is the lower function, $\beta$ the upper function.

This boundary value problem is replaced by another one, which is dependent on the parameter $M \in\left(M_{0},+\infty\right), M_{0}>0$,

$$
\begin{aligned}
& \left(\varphi_{M}\left(t, x, x^{\prime}\right)\right)^{\prime}=f_{M}\left(t, x, x^{\prime}\right), \quad t \in I=[a, b], \\
& H_{1} x=h_{1}, \quad H_{2} x=h_{2}, \quad \alpha \leq x \leq \beta,
\end{aligned}
$$

where $\varphi_{M} \in C\left(I \times R^{2}, R\right)$ is strictly increasing in $x^{\prime}$ for fixed $t$ and $x$, and $f: I \times R^{2} \rightarrow R$ satisfies the Caratheodory conditions.

Definition 1 A function $x \in C^{1}(I, R)$ is a solution of (1), if $\varphi\left(t, x(t), x^{\prime}(t)\right)$ is absolutely continuous on $I$ and (1) is satisfied almost everywhere on $I$.

We provide below definitions of generalized upper and lower functions and the generalized solution along with Theorem 1 from [1-3]. This is needed to prove the main result.

Definition 2 The class $B B^{+}(I, R)$ consists of functions $\alpha: I \rightarrow R$, which possess the property: for any $t \in(a, b]$ there exist the left derivative $\alpha_{l}^{\prime}(t)$ and the $\operatorname{limit}_{\lim _{\tau \rightarrow t-}} \alpha_{l}^{\prime}(\tau)$, and

○2014 Lepin; licensee Springer. This is an Open Access article distributed under the terms of the Creative Commons Attribution License (http://creativecommons.org/licenses/by/2.0), which permits unrestricted use, distribution, and reproduction in any medium, provided the original work is properly cited. 
$\alpha_{l}^{\prime}(t) \geq \lim _{\tau \rightarrow t-} \alpha_{l}^{\prime}(\tau)$; for any $t \in[a, b)$ there exist the right derivative $\alpha_{r}^{\prime}(t)$ and the limit $\lim _{\tau \rightarrow t_{+}} \alpha_{r}^{\prime}(\tau)$, and $\alpha_{r}^{\prime}(t) \leq \lim _{\tau \rightarrow t+} \alpha_{r}^{\prime}(\tau)$, and, for any $t \in(a, b), \alpha_{l}^{\prime}(t) \leq \alpha_{r}^{\prime}(t)$.

The class $B B^{-}(I, R)$ consists of functions $\beta: I \rightarrow R$, which possess the following property: for any $t \in(a, b]$ there exist the left derivative $\beta_{l}^{\prime}(t)$ and the $\operatorname{limit}_{\lim _{\tau \rightarrow t-}} \beta_{l}^{\prime}(\tau)$, and $\beta_{l}^{\prime}(t) \leq \lim _{\tau \rightarrow t-} \beta_{l}^{\prime}(\tau)$; for any $t \in[a, b)$ there exist the right derivative $\beta_{r}^{\prime}(t)$ and the limit $\lim _{\tau \rightarrow t+} \beta_{r}^{\prime}(\tau)$, and $\beta_{r}^{\prime}(t) \geq \lim _{\tau \rightarrow t+} \beta_{r}^{\prime}(\tau)$, and, for any $t \in(a, b), \beta_{l}^{\prime}(t) \geq \beta_{r}^{\prime}(t)$.

Definition 3 We call a bounded function $\alpha \in B B^{+}(I, R)$ a generalized lower function and write $\alpha \in A G(I, R)$, if in any interval $[c, d] \in I$, where this function satisfies the Lipschitz condition, for any $t_{1} \in(c, d)$ and $t_{2} \in\left(t_{1}, d\right)$ where the derivative exists, the inequality

$$
\varphi\left(t_{2}, \alpha\left(t_{2}\right), \alpha^{\prime}\left(t_{2}\right)\right)-\varphi\left(t_{1}, \alpha\left(t_{1}\right), \alpha^{\prime}\left(t_{1}\right)\right) \geq \int_{t_{1}}^{t_{2}} f\left(s, \alpha(s), \alpha^{\prime}(s)\right) d s
$$

holds. We will call a bounded function $\beta \in B B^{-}(I, R)$ a generalized upper function and write $\beta \in B G(I, R)$, if in any interval $[c, d] \in I$, where this function satisfies the Lipschitz condition, for any $t_{1} \in(c, d)$ and $t_{2} \in\left(t_{1}, d\right)$ where the derivative exists, the inequality

$$
\varphi\left(t_{2}, \beta\left(t_{2}\right), \beta^{\prime}\left(t_{2}\right)\right)-\varphi\left(t_{1}, \beta\left(t_{1}\right), \beta^{\prime}\left(t_{1}\right)\right) \leq \int_{t_{1}}^{t_{2}} f\left(s, \beta(s), \beta^{\prime}(s)\right) d s
$$

holds.

A function $x: I \rightarrow R$ will be called a generalized solution, if $x \in A G(I, R) \cap B G(I, R)$.

A generalized solution has a derivative at any point, possibly infinite, either $-\infty$ or $+\infty$, and $x^{\prime}$ is continuous on $[-\infty,+\infty]$; if in some interval the derivative $x^{\prime}$ does not attain the values $-\infty$ or $+\infty$, then $x$ is a solution of (1) in this interval.

Theorem 1 Let $\alpha \in A G(I, R), \beta \in B G(I, R)$ and $\alpha \leq \beta$. Then for any $A \in[\alpha(a), \beta(a)]$ and $B \in[\alpha(b), \beta(b)]$ there exists a generalized solution of the Dirichlet problem

$$
\left(\varphi\left(t, x, x^{\prime}\right)\right)^{\prime}=f\left(t, x, x^{\prime}\right), \quad x(a)=A, \quad x(b)=B, \quad \alpha \leq x \leq \beta .
$$

In addition to conditions on $\alpha$ and $\beta$ the compactness conditions are needed for solvability of the boundary value problem (1)-(2). The Nagumo condition [4] for $\varphi$-Laplacian and the Schrader condition [5] are sufficient conditions for compactness of a set of solutions. We accept the following compactness conditions.

Definition 4 We say that the compactness condition is fulfilled, if for all $A \in[\alpha(a), \beta(a)]$ and $B \in[\alpha(b), \beta(b)]$ any generalized solution of the Dirichlet problem (4) is a solution.

It is clear that this condition is weaker than the Schrader condition. A set of solutions of the Dirichlet problem (4) will be denoted by $S$.

Remark 1 If $\alpha \in A G(I, R), \beta \in B G(I, R), \alpha \leq \beta$ and the compactness condition is fulfilled, then the Dirichlet problem (4) has a solution. 
Theorem 2 Let $\alpha \in A G(I, R), \beta \in B G(I, R)$ and the compactness condition be fulfilled. If the boundary value problem (3) has a solution $u_{M}$ for all $M \in\left(M_{0},+\infty\right)$ and for $t \in I$

$$
\varphi_{M}\left(t, x, x^{\prime}\right)=\varphi\left(t, x, x^{\prime}\right), \quad f_{M}\left(t, x, x^{\prime}\right)=f\left(t, x, x^{\prime}\right), \quad \alpha \leq x \leq \beta,\left|x^{\prime}\right| \leq M,
$$

then there exists $M_{1} \in\left(M_{0},+\infty\right)$ such that $u_{M_{1}}$ solves the boundary value problem (1)-(2).

Proof Notice that the results in [6] imply that $\sup \left\{\left\|x^{\prime}\right\|_{C}: x \in S\right\}=M_{0}<+\infty$. Suppose the contrary. Let the sequence $\left\{M_{i}\right\}$, where $M_{i} \in\left(M_{0},+\infty\right), i=1,2, \ldots$ tend to infinity. Consider the sequence $\left\{u_{i}\right\}$, where $u_{i}=u_{M_{i}}, i=1,2, \ldots$. We can assume, without loss of generality, that it converges in any rational points of the interval $(a, b)$ to the function $u$, located between $\alpha$ and $\beta$. Notice that without loss of generality for any interval $\left(a_{1}, b_{1}\right) \subset(a, b)$ it follows from the boundedness of $u$ and the Mean Value Formula that there exists an interval $[c, d] \subset\left(a_{1}, b_{1}\right)$ such that

$$
\sup \left\{\left|u_{i}^{\prime}(t)\right|: i \in\{1,2, \ldots\}, t \in[c, d]\right\}=L<+\infty \text {. }
$$

It is clear that $u_{i}, i \in\{1,2, \ldots\}$, and $u$ satisfy the Lipschitz condition with constant $L$ in $[c, d]$. The $u$ can be extended by continuity to the entire interval $[c, d]$, and thus we obtain a function $u$ that satisfies the Lipschitz condition. It follows from the Lipschitz condition that $\left\{u_{i}(t)\right\}$ converges to $u(t)$ for any $t \in[c, d]$. It is clear that the derivatives $\left\{u_{i}^{\prime}(t)\right\}$ converge to the derivative $u^{\prime}(t)$ for any $t \in[c, d]$. Therefore, $u(t)$ is a solution of (1) in the interval $[c, d]$. Continuing the construction of $u(t)$ on both sides, one gets a solution of (1) on the maximal interval $\left(c_{1}, d_{1}\right)$. If $c_{1}>a$, then $\lim _{t \rightarrow c_{1}+} u^{\prime}(t)$ is either $-\infty$ or $+\infty$. Similarly, if $d_{1}<b$, then $\lim _{t \rightarrow d_{1}-} u^{\prime}(t)$ is either $-\infty$ or $+\infty$. If $c_{1}=a$ and $\lim _{t \rightarrow a_{+}} u^{\prime}(t)$ is not $-\infty$ or $+\infty$, then $u(t)$ can be continued to $a$. Similarly, if $d_{1}=b$ and $\lim _{t \rightarrow b-} u^{\prime}(t)$ is not $-\infty$ nor $+\infty$, then $u(t)$ can be continued to $b$. By repeating this construction, find an open set $I_{1}$ in $I$, where the function $u(t)$ is defined and $u(t)$ is a solution of $(1)$ on intervals from $I_{1}$. A set $I_{2}=I \backslash I_{1}$ is closed and nowhere dense. For $t \in I_{2}$ the $\operatorname{limit}_{\lim _{i \rightarrow \infty}} u_{i}^{\prime}(t)$ is equal to $-\infty$ or $+\infty$. Indeed, assuming the contrary and acting as above, we get $t \in I_{1}$. Extend $u(t)$ to irrational points of $I_{2}$. If $a \in I_{2}$, then $u(a)=\lim _{t \rightarrow a+} u(t)$, and in the remaining cases $u(\tau)=$ $\lim _{t \rightarrow \tau-} u(t)$. The above limits exist since $u(t)$ is monotone in neighborhood of any point from $I_{2}$. Similarly we get for $t \in I_{2}, u^{\prime}(t)=\lim _{i \rightarrow \infty} u_{i}^{\prime}(t)$ and $\lim _{\tau \rightarrow t} u^{\prime}(\tau)=u^{\prime}(t)$. Therefore $u(t)$ is a generalized solution of (1). It follows from the compactness condition that $u(t)$ is a solution of (1). Let us show that the sequence $\left\{u_{i}^{\prime}(t)\right\}$ uniformly converges to $u^{\prime}(t)$. Suppose the contrary is true. We assume, without loss of generality, that there exist $\varepsilon>0$ and a sequence $\left\{t_{i}\right\}$, where $t_{i} \in I, i=1,2, \ldots$ such that $\left|u^{\prime}\left(t_{i}\right)-u_{i}^{\prime}\left(t_{i}\right)\right|>\varepsilon, i=1,2, \ldots$ and $\lim _{i \rightarrow \infty} t_{i}=t_{0}$. Consider the case $u_{i}^{\prime}\left(t_{i}\right)>u^{\prime}\left(t_{i}\right)+\varepsilon, i=1,2, \ldots$ We can assume, without loss of generality, that $u_{i}^{\prime}\left(t_{0}\right)>u^{\prime}\left(t_{0}\right)+\varepsilon / 2, i=1,2, \ldots$, and this contradicts the equality $\lim _{i \rightarrow \infty} u_{i}^{\prime}\left(t_{0}\right)=u^{\prime}\left(t_{0}\right)$. The uniform convergence is proved. We can conclude now that all $u_{i}(t)$ are the solutions of the boundary value problem (1)-(2).

Remark 2 Theorem 2 gives the possibility to prove the solvability of boundary value problems if the solvability of more simple boundary value problems is known.

Remark 3 If $\alpha^{\prime}(a) \geq \beta^{\prime}(a)$ and the inequalities $\alpha^{\prime}(a) \geq x^{\prime}(a) \geq \beta^{\prime}(a)$ hold for a solution $x$ of the boundary value problem (1)-(2), then the compactness condition (Definition 4) can be weakened. 
Definition 5 We will say that the compactness condition holds if for any $A_{1} \in\left[\beta^{\prime}(a), \alpha^{\prime}(a)\right]$ and $B \in[\alpha(b), \beta(b)]$ all generalized solutions of the problem

$$
\left(\varphi\left(t, x, x^{\prime}\right)\right)^{\prime}=f\left(t, x, x^{\prime}\right), \quad x^{\prime}(a)=A_{1}, \quad x(b)=B, \quad \alpha \leq x \leq \beta,
$$

are classical solutions.

Example One way to use Theorem 2 is to verify that for all $t \in I, x, x^{\prime} \in R$ and $M \in$ $\left(M_{0},+\infty\right), M_{0}>0$, the following conditions are satisfied:

$$
\begin{aligned}
\varphi_{M}\left(t, x, x^{\prime}\right) & =\varphi\left(t, x, x^{\prime}\right), \\
f_{M}\left(t, x, x^{\prime}\right) & =f\left(t, x, \delta\left(-M, x^{\prime}, M\right)\right),
\end{aligned}
$$

where $\delta(u, v, w)=u$ if $v<u, \delta(u, v, w)=v$ if $u \leq v \leq w, \delta(u, v, w)=w$ if $w<v$.

\section{Competing interests}

The author declares that he has no competing interests.

\section{Authors' contributions}

The author participated in drafting, revising and commenting on the manuscript. The author read and approved the final manuscript.

\section{Acknowledgements}

The author sincerely thanks the reviewers for their valuable suggestions and useful comments. This research was supported by the Institute of Mathematics and Computer Science, University of Latvia.

Received: 13 December 2013 Accepted: 28 April 2014 Published: 13 May 2014

\section{References}

1. Lepin, LA: Generalized solutions and solvability of boundary value problems for the second order differential equations. Differ. Equ. 18, 1323-1330 (1982)

2. Lepin, AY, Lepin, LA: Boundary Value Problems for Ordinary Differential Equations. Zinatne, Riga (1988) (Russian)

3. Lepin, AY, Lepin, LA: Generalized lower and upper functions for $\varphi$-Laplacian equations. Differ. Equ. (2014, in press)

4. Nagumo, M: Über die Differentialgleichung $y^{\prime \prime}=f\left(x, y, y^{\prime}\right)$. Proc. Phys. Math. Soc. Jpn. 19(3), 861-866 (1937)

5. Schrader, KW: Existence theorems for second order boundary value problems. J. Differ. Equ. 5(3), 572-584 (1969)

6. Lepin, AY: Compactness of generalized solutions between the generalized lower and upper functions. Proc. LUMII Math. Differ. Equ. 11, 22-24 (2011)

\section{Submit your manuscript to a SpringerOpen ${ }^{\ominus}$ journal and benefit from:}

- Convenient online submission

Rigorous peer review

- Immediate publication on acceptance

- Open access: articles freely available online

- High visibility within the field

- Retaining the copyright to your article 